\title{
Imprimitive cometric association schemes: Constructions and analysis
}

\author{
William J. Martin • Mikhail Muzychuk • \\ Jason Williford
}

Received: 31 December 2005 / Accepted: 25 September 2006 /

Published online: 9 January 2007

(C) Springer Science + Business Media, LLC 2007

\begin{abstract}
Dualizing the "extended bipartite double" construction for distance-regular graphs, we construct a new family of cometric (or $Q$-polynomial) association schemes with four associate classes based on linked systems of symmetric designs. The analysis of these new schemes naturally leads to structural questions concerning imprimitive cometric association schemes, some of which we answer with others being left as open problems. In particular, we prove that any $Q$-antipodal association scheme is dismantlable: the configuration induced on any subset of the equivalence classes in the $Q$-antipodal imprimitivity system is again a cometric association scheme. Further examples are explored.
\end{abstract}

Keywords Association scheme $\cdot$ Cometric $\cdot Q$-polynomial $\cdot$ Imprimitive $\cdot$ Spherical design $\cdot$ Linked system of symmetric designs

\section{A census of cometric association schemes}

Very few examples are known of association schemes that are cometric but not metric. Much effort has been devoted to the study and classification of $Q$-polynomial

Dedicated to the memory of Dom de Caen, 1956 - 2002.

\section{W. J. Martin $(\bowtie)$}

Department of Mathematical Sciences and Department of Computer Science, Worcester Polytechnic Institute, Worcester, Massachusetts

e-mail: martin@wpi.edu

\section{Muzychuk}

Department of Computer Science and Mathematics, Netanya Academic College,

Netanya 42365 Israel

e-mail: muzy@netanya.ac.il

J. Williford

Department of Mathematical Sciences, Worcester Polytechnic Institute, Worcester, Massachusetts e-mail: jsw@wpi.edu 
distance-regular graphs, i.e., association schemes that are both metric and cometric, since the most important families of distance-regular graphs enjoy the cometric property. (See $[1,2,18]$ for a good starting point in the substantial literature on this subject.) The following list gives all examples of cometric association schemes known to us (see [15] for details on spherical designs):

- $Q$-polynomial distance-regular graphs (this includes all symmetric 2-class schemes);

- duals of metric translation schemes (e.g., the subscheme induced on even-weight codewords in the perfect binary Golay code);

- bipartite doubles of Hermitian forms dual polar spaces $\left[{ }^{2} A_{2 d-1}(r)\right]$ [1, p. 315] (see Section 3);

- schemes arising from linked systems of symmetric designs (one infinite family known [5], 3-class, $Q$-antipodal);

- the extended $Q$-bipartite doubles of the above infinite family (4-class, $Q$-bipartite and $Q$-antipodal);

- the block schemes of the Witt designs 4-(11,5,1), 5-(24,8,1) and a 4-(47,11,8) design arising from a quadratic residue code [9] (these are primitive 3-class schemes on 66, 759 and 4324 vertices, respectively);

- the block schemes of the 5-(12,6,1) design and the 5-(24,12,48) design arising from the extended Golay codes (these are $Q$-bipartite 4-class schemes on 132 and 2576 vertices, respectively);

- the scheme determined by the shortest vectors in the Leech lattice, a tight spherical 11 -design [10] in $\mathbb{R}^{24}$ (6-class, $Q$-bipartite, 196560 vertices) and five schemes carried on subsets of this vertex set obtained by choosing only those points lying on certain affine subspaces of codimension one or two (each of these spherical designs is a "derived spherical design" of this one or of one of the others):

- a 3-class primitive scheme on 2025 vertices

- two 4-class $Q$-bipartite schemes on 2816 and 4600 vertices

- a 4-class primitive scheme on 7128 vertices

- a 5-class primitive scheme on 47104 vertices;

- three 4-class $Q$-bipartite schemes carried on the shortest vectors of the lattices $E_{6}$, $E_{7}$ and $E_{8}$ (72 vertices, 126 vertices, and 240 vertices, respectively) - this last one corresponds to the Gosset polytope, a spherical 7-design [10] in $\mathbb{R}^{8}$;

- the scheme on the vertices of the 24-cell, a spherical 5-design in $\mathbb{R}^{4}$ (4-class, $Q$-bipartite, $Q$-antipodal, 24 vertices);

- the antipodal quotient of the Leech lattice example (3-class, primitive, 98280 vertices);

- the shortest vectors of a lattice in $\mathbb{R}^{16}$ (4-class, $Q$-bipartite, 512 vertices), this is the overlattice of the Barnes-Wall lattice;

- a scheme arising from 275 lines through the origin in $\mathbb{R}^{22}$ with only two angles among them, a spherical 5-design (5-class, $Q$-bipartite and $Q$-antipodal, 550 vertices);

- the bipartite double of the above scheme on 47104 vertices (11-class, $Q$-bipartite, 94208 vertices); 
- three $Q$-antipodal schemes constructed below by "dismantling" larger schemes related to the Golay codes (a 4-class on 162 vertices, a 5-class on 486 vertices, and a 6-class on 1536 vertices).

(All parameters for the exceptional schemes listed above can be found on the web at http: //users . wpi . edu/ martin/RESEARCH/QPOL/)

As we consider this list, we are reminded of the following

Conjecture 1.1 (Bannai/Ito [1, p. 312]). Every primitive cometric scheme of sufficiently large diameter is metric as well.

\subsection{Outline of the paper}

The balance of the paper is structured as follows. In Section 2, we review the basic definitions, introduce our notation and consider Suzuki's Theorem on imprimitive cometric association schemes. In Section 3, we introduce the extended $Q$-bipartite double of a cometric association scheme. We discuss several known examples which can be cast in this language and then construct a new infinite family of 4-class imprimitive cometric schemes based on linked systems of symmetric designs, which we briefly review.

Section 4 considers some structural questions about $Q$-bipartite and $Q$-antipodal schemes. First, we review a result of Brouwer et al., showing that a $Q$-bipartite scheme is necessarily an index two cover of its natural quotient. We derive some parameter restrictions from this simple result. We conjecture that the number of equivalence classes in any $Q$-antipodal imprimitivity system is bounded above by the first multiplicity in the $Q$-polynomial ordering and we prove this when $d$ is odd. Two corollaries follow, giving information about the graphs in such an association scheme. Finally, we prove our main result which states that every $Q$-antipodal association scheme is dismantlable: the configuration induced on any subset of $w^{\prime}$ of the equivalence classes in the $Q$-antipodal imprimitivity system is again a cometric association scheme, this scheme being again $Q$-antipodal provided $w^{\prime}>1$. We then use this result to give a new proof of a theorem of van Dam which characterizes linked systems of symmetric designs and we also construct new $Q$-antipodal association schemes from known ones.

\section{Background material}

Let $(X, \mathbf{A})$ be a symmetric $d$-class association scheme $[1,2,13]$ on $v$ vertices with Schur idempotents $\mathbf{A}=\left\{A_{0}, \ldots, A_{d}\right\}$, primitive (ordinary) idempotents $E_{0}, \ldots, E_{d}$, eigenmatrices $P$ and $Q=v P^{-1}$, valencies $k_{i}$ and multiplicities $m_{j}$. When we say $(X, \mathbf{A})$ is cometric (or $Q$-polynomial), we imply that the ordering $E_{0}, E_{1}, \ldots, E_{d}$ is a $Q$-polynomial ordering. That is, the Krein parameters $q_{i j}^{k}$ given by

$$
E_{i} \circ E_{j}=\frac{1}{v} \sum_{k=0}^{d} q_{i j}^{k} E_{k}
$$


(where $\circ$ denotes Schur-or entrywise-multiplication) satisfy

- $q_{i j}^{k}=0$ whenever $k<|i-j|$ or $k>i+j$, and

- $q_{i j}^{i+j}>0$ whenever $i+j \leq d$.

The parameters of a cometric scheme $(X, \mathbf{A})$ are entirely determined by its Krein array

$$
\iota^{*}(X, \mathbf{A})=\left\{b_{0}^{*}, b_{1}^{*}, \ldots, b_{d-1}^{*} ; c_{1}^{*}, c_{2}^{*}, \ldots, c_{d}^{*}\right\}
$$

where $b_{j}^{*}=q_{1, j+1}^{j}, c_{j}^{*}=q_{1, j-1}^{j}$ and we also define $a_{j}^{*}=q_{1 j}^{j}=m-b_{j}^{*}-c_{j}^{*}$ where, here and below, we write $m$ instead of $m_{1}$.

A cometric scheme is $Q$-bipartite if all $a_{j}^{*}=0$. This is equivalent to the condition that $q_{i j}^{k}=0$ whenever $i+j+k$ is odd. A cometric scheme is $Q$-antipodal if $b_{j}^{*}=c_{d-j}^{*}$ for all $j$ except possibly $j=\left\lfloor\frac{d}{2}\right\rfloor$.

An association scheme is imprimitive if some graph in the scheme is disconnected. In the next theorem and henceforth, we will let $I_{r}$ (or simply $I$ ) denote the $r \times r$ identity matrix and $J_{r}$ (or $J$ ) will denote the all ones matrix of order $r \times r$.

Theorem 2.1 (see [1, 2, 13]). The following are equivalent:

(i) $(X, \mathbf{A})$ is imprimitive;

(ii) for some $j>0, E_{j}$ has repeated columns;

(iii) for some subset $\mathcal{I}=\left\{i_{0}=0, i_{1}, \ldots, i_{s}\right\}$ of $\{0,1, \ldots, d\}$ and some ordering of the vertices $\sum_{h=0}^{s} A_{i_{h}}=I_{w} \otimes J_{r}$ for some integers $w$ and $r$ with $v=w r, 1<$ $w, r<v$;

(iv) for some subset $\mathcal{J}=\left\{j_{0}=0, j_{1}, \ldots, j_{s}\right\}$ of $\{0,1, \ldots, d\}$ and some ordering of the vertices $\sum_{h=0}^{s} E_{j_{h}}$ has form $\frac{1}{r}\left(I_{w} \otimes J_{r}\right)$ for some integers $w$ and $r$ with $v=w r, 1<w, r<v$.

The same scheme may have several such imprimitivity systems and our language must distinguish them; for example, the vertex set of a scheme which is $Q$-antipodal admits a partition into " $Q$-antipodal classes" and a scheme which is $Q$-bipartite has a " $Q$-bipartite imprimitivity system" which partitions the vertices into "dual bipartite classes". In each case, we will use $r$ for the size of a class and $w=v / r$ for the number of such classes in this partition. We will also continue to use $\mathcal{I}$ for the set of indices $0 \leq i \leq d$ for which $A_{i}$ has all zeros on blocks indexed by distinct classes and $\mathcal{J}$ for the set of indices $0 \leq j \leq d$ for which $E_{j}$ has all columns indexed by any class identical.

Theorem 2.2 (Suzuki [17]). Suppose (X, A) is an imprimitive cometric association scheme. Then one of the following holds:

- $(X, \mathbf{A})$ is $Q$-bipartite and $\mathcal{J}=\{0,2,4, \ldots\}$;

- $(X, \mathbf{A})$ is $Q$-antipodal and $\mathcal{J}=\{0, d\}$;

- $d=4, \iota^{*}(X, \mathbf{A})=\left\{m, m-1,1, b_{3}^{*} ; 1, c_{2}^{*}, m-b_{3}^{*}, 1\right\}$ and $\mathcal{J}=\{0,3\}$;

- $d=6, \iota^{*}(X, \mathbf{A})=\left\{m, m-1,1, b_{3}^{*}, b_{4}^{*}, 1 ; 1, c_{2}^{*}, m-b_{3}^{*}, 1, c_{5}^{*}, m\right\}$ (where $a_{2}^{*}=$ $\left.a_{4}^{*}+a_{5}^{*}\right)$ and $\mathcal{J}=\{0,3,6\}$. 
Very recently, Cerzo and Suzuki [7] have shown that there are no association schemes of the third type in the list. No examples are known of the last type.

We mention an earlier result of Dickie whose proof uses the same ideas as those used in the proof of the above theorem.

Theorem 2.3 (Dickie [11]). In a cometric scheme, if $a_{1}^{*} \neq 0$, then $a_{j}^{*} \neq 0$ for all $j<d$.

If $(X, \mathbf{A})$ is cometric with $Q$-polynomial ordering $0,1, \ldots, d$, then the entries in column 1 of the matrix $Q$ are all distinct and we may define a natural ordering on the relations by the requirement that $Q_{01}>Q_{11}>\cdots>Q_{d 1}$. We will use this throughout the paper.

\section{The extended $Q$-bipartite double}

In this section, we give a construction dual to the "extended bipartite double" construction of [2, Sec. 1.11] and show how this gives rise to a new infinite family of 4-class cometric association schemes.

Let us first review the bipartite double of an arbitrary association scheme. If we begin with any scheme with associate matrices $A_{i}$ and primitive idempotents $E_{j}(0 \leq i, j \leq d)$, then the bipartite double has associate matrices

$$
A_{i}^{+}=\left[\begin{array}{cc}
A_{i} & 0 \\
0 & A_{i}
\end{array}\right] \quad \text { and } \quad A_{i}^{-}=\left[\begin{array}{cc}
0 & A_{i} \\
A_{i} & 0
\end{array}\right]
$$

and primitive idempotents

$$
E_{j}^{+}=\frac{1}{2}\left[\begin{array}{ll}
E_{j} & E_{j} \\
E_{j} & E_{j}
\end{array}\right] \quad \text { and } \quad E_{j}^{-}=\frac{1}{2}\left[\begin{array}{cc}
E_{j} & -E_{j} \\
-E_{j} & E_{j}
\end{array}\right] .
$$

A cometric scheme $(X, \mathbf{A})$ is almost dual bipartite if $a_{j}^{*}=0$ for $j<d$ but $a_{d}^{*} \neq 0$. (These schemes admit a second $Q$-polynomial ordering of their idempotents.) Bannai and Ito [1, p. 315] proved that the bipartite double of an almost dual bipartite cometric scheme is cometric as well, with $Q$-polynomial ordering $E_{0}^{+}, E_{1}^{-}, E_{2}^{+}, E_{3}^{-}, \ldots, E_{0}^{-}$. As stated in the introduction, the Hermitian forms dual polar space graphs $\left[{ }^{2} A_{2 d-1}(r)\right]$ give an infinite family of examples, with arbitrarily large diameter, where this bipartite double is cometric but not metric. In this section, we examine a related construction (Theorem 3.1(i)), called the extended Q-bipartite double.

Theorem 3.1. Let $(X, \mathbf{A})$ be a d-class cometric association scheme on $v$ vertices with primitive idempotents $E_{j}$ and Krein parameters $a_{j}^{*}, b_{j}^{*}, c_{j}^{*}$ satisfying $b_{j}^{*}+c_{j+1}^{*}=$ $m+1$ for $0 \leq j<d$. Then

(i) there exists an association scheme $(\hat{X}, \hat{\mathbf{A}})$ on $2 v$ vertices where $\hat{X}=X \times\{0,1\}$ and

$$
\hat{\mathbf{A}}=\left\{A_{0}^{+}, A_{1}^{+}+A_{d}^{-}, A_{2}^{+}+A_{d-1}^{-}, \ldots, A_{0}^{-}\right\}
$$


where $A_{0}, A_{1}, A_{2}, \ldots$ is the natural ordering of Schur idempotents in the original scheme. Moreover, this is a Q-bipartite cometric scheme with Q-polynomial ordering

$$
E_{0}^{+}, E_{0}^{-}+E_{1}^{-}, E_{1}^{+}+E_{2}^{+}, E_{2}^{-}+E_{3}^{-}, \ldots, E_{d}^{ \pm}
$$

where the last matrix is $E_{d}^{+}$if $d$ is odd and $E_{d}^{-}$if $d$ is even;

(ii) the idempotent $E_{1}+E_{2}$ generates a cometric fusion scheme $(X, \overline{\mathbf{A}})$ of the original scheme $(X, \mathbf{A})$; this is the $Q$-bipartite quotient of the scheme $(\hat{X}, \hat{\mathbf{A}})$.

Proof: From the viewpoint of $C$-algebras, the proof of (i) is equivalent to a proof of Theorem 1.11.2(vi) in [2] and follows from direct computation:

$$
\begin{aligned}
& \left(E_{0}^{-}+E_{1}^{-}\right) \circ\left(E_{j}^{+}+E_{j+1}^{+}\right) \\
& =\frac{1}{2 v}\left[b_{j-1}^{*} E_{j-1}^{-}+\left(a_{j}^{*}+b_{j}^{*}+1\right) E_{j}^{-}+\left(a_{j+1}^{*}+c_{j+1}^{*}+1\right) E_{j+1}^{-}+c_{j+2}^{*} E_{j+2}^{-}\right] \\
& =\frac{1}{2 v}\left[b_{j-1}^{*}\left(E_{j-1}^{-}+E_{j}^{-}\right)+c_{j+2}^{*}\left(E_{j+1}^{-}+E_{j+2}^{-}\right)\right] .
\end{aligned}
$$

Similarly,

$$
\begin{aligned}
\left(E_{0}^{-}+E_{1}^{-}\right) \circ\left(E_{j}^{-}+E_{j+1}^{-}\right) & =\frac{1}{2 v}\left[b_{j-1}^{*}\left(E_{j-1}^{+}+E_{j}^{+}\right)+c_{j+2}^{*}\left(E_{j+1}^{+}+E_{j+2}^{+}\right)\right] \\
\left(E_{0}^{-}+E_{1}^{-}\right) \circ\left(E_{d-1}^{ \pm}+E_{d}^{ \pm}\right) & =\frac{1}{2 v}\left[b_{d-2}^{*}\left(E_{d-2}^{\mp}+E_{d-1}^{\mp}\right)+(m+1) E_{d}^{\mp}\right] \\
\left(E_{0}^{-}+E_{1}^{-}\right) \circ E_{d}^{ \pm} & =\frac{1}{2 v} b_{d-1}^{*}\left(E_{d-1}^{\mp}+E_{d}^{\mp}\right) .
\end{aligned}
$$

The proof of (ii) follows by looking at the upper-lefthand block of each matrix on each side of the above identities.

Remark 3.2. Without proof, we note that the Krein array for the fusion scheme in part (ii) is

$$
\iota^{*}(X, \hat{\mathbf{A}})=\left\{m_{1}+m_{2}, \frac{b_{1}^{*} b_{2}^{*}}{c_{2}^{*}}, \frac{b_{3}^{*} b_{4}^{*}}{c_{2}^{*}}, \ldots, \frac{b_{d-2}^{*} b_{d-1}^{*}}{c_{2}^{*}} ; 1, \frac{c_{3}^{*} c_{4}^{*}}{c_{2}^{*}}, \frac{c_{5}^{*} c_{6}^{*}}{c_{2}^{*}}, \ldots, \frac{c_{d}^{*}\left(m_{1}+1\right)}{c_{2}^{*}}\right\}
$$

when $d$ is odd and

$$
\iota^{*}(X, \hat{\mathbf{A}})=\left\{m_{1}+m_{2}, \frac{b_{1}^{*} b_{2}^{*}}{c_{2}^{*}}, \frac{b_{3}^{*} b_{4}^{*}}{c_{2}^{*}}, \ldots, \frac{b_{d-3}^{*} b_{d-2}^{*}}{c_{2}^{*}} ; 1, \frac{c_{3}^{*} c_{4}^{*}}{c_{2}^{*}}, \frac{c_{5}^{*} c_{6}^{*}}{c_{2}^{*}}, \ldots, \frac{c_{d-1}^{*} c_{d}^{*}}{c_{2}^{*}}\right\}
$$

when $d$ is even.

Example 3.3. If $(X, \mathbf{A})$ is the association scheme of a strongly regular graph with $b_{1}^{*}+c_{2}^{*}=m+1$, then the extended $Q$-bipartite double is again a metric scheme. This 黑Springer 
is a distance-regular antipodal double cover of a complete graph, the complete graph being the scheme arising in part (ii) of the theorem. This construction is well-known; it works precisely when $v=2(2 k-\lambda-\mu)$, i.e., when the given strongly regular graph belongs to the switching class of a regular two-graph [2, Theorem 1.5.6].

Example 3.4 (A. Munemasa, personal communication). The Soicher graph $\Sigma$ for $M_{22}: 2$ is a distance-regular graph of diameter three having intersection array $\iota(\Sigma)=$ $\{110,81,12 ; 1,18,90\}$. The underlying association scheme $(X, \mathbf{A})$ is cometric with Krein array $\iota^{*}(X, \mathbf{A})=\{55,49,21 ; 1,7,35\}$ so Theorem 3.1(i) applies. We then obtain a 4-class scheme which is both metric and cometric. This distance-regular graph was discovered by Meixner and has intersection array $\{176,135,24,1 ; 1,24,135,176\}$. If $A_{0}, A_{1}, A_{2}, A_{3}$ are the distance matrices of the Soicher graph, then Munemasa observed that the adjacency matrix of the Meixner graph can be expressed as

$$
A_{1}^{+}+A_{3}^{-}=\left[\begin{array}{ll}
A_{1} & A_{3} \\
A_{3} & A_{1}
\end{array}\right]
$$

as in part (i) of the theorem.

Several open parameter sets for diameter three cometric distance-regular graphs also satisfy the conditions of Theorem 3.1. These include

$$
\begin{array}{rrr}
v & =322 & \{60,45,8 ; 1,12,50\} \\
v & =392 & \{69,56,10 ; 1,14,60\} \\
v & =378 & \{78,50,9 ; 1,15,60\} \\
v & =800 & \{119,100,15 ; 1,20,105\} \\
v & =900 & \{174,110,18 ; 1,30,132\} .
\end{array}
$$

Example 3.5. The block scheme of the 4-(11,5,1) Witt design is a cometric scheme with Krein array $\iota^{*}(X, \mathbf{A})=\left\{10, \frac{242}{27}, \frac{11}{5} ; 1, \frac{55}{27}, \frac{44}{5}\right\}$. Clearly the conditions of Theorem 3.1(i) are met. But the extended $Q$-bipartite double of this scheme is already well-known: it is the block scheme of the 5-(12,6,1) Witt design with Krein array $\iota^{*}(X, \mathbf{A})=\left\{11,10, \frac{242}{27}, \frac{11}{5} ; 1, \frac{55}{27}, \frac{44}{5}, 11\right\}$. By the same token, the induced association scheme on the even subcode of the perfect binary Golay code (i.e., the dual scheme of the coset graph of the perfect code), with $\operatorname{Krein}$ array $\iota^{*}(X, \mathbf{A})=\{23,22,21 ; 1,2,3\}$, has as its extended $Q$-bipartite double the induced scheme on the extended binary Golay code with Krein array $\iota^{*}(X, \mathbf{A})=\{24,23,22,21 ; 1,2,3,24\}$.

\subsection{Linked systems of symmetric designs}

Our interest for the remainder of this section is to apply part (i) of Theorem 3.1 to the association schemes of linked systems of symmetric designs, which we now briefly review. (See $[5,14,16]$ for information on these structures.)

A linked system of $\ell$ symmetric $(v, k, \lambda)$ designs is a graph $G$ defined on a vertex set

$$
X=\mathcal{P}_{1} \cup \mathcal{P}_{2} \cup \cdots \cup \mathcal{P}_{\ell+1}
$$


where $\pi=\left\{\mathcal{P}_{1}, \mathcal{P}_{2}, \ldots, \mathcal{P}_{\ell+1}\right\}$ is a partition of $X$ into $\ell+1$ sets of size $v$ each, enjoying the following properties:

1. partition $\pi$ is a proper coloring of $G$ : no edge of $G$ has both ends in the same class $\mathcal{P}_{i}$

2. for any $i \neq j$, the subgraph of $G$ induced on $\mathcal{P}_{i} \cup \mathcal{P}_{j}$ is the incidence graph of a symmetric $(v, k, \lambda)$ design;

3. for any three distinct classes $\mathcal{P}_{i}, \mathcal{P}_{j}, \mathcal{P}_{k}$, the number of common neighbors of a vertex $x$ in $\mathcal{P}_{i}$ and a vertex $y$ in $\mathcal{P}_{j}$ which lie in $\mathcal{P}_{k}$ depends only on whether $x$ and $y$ are adjacent in $G$ or not; it does not depend on the choice of $x$ and $y$ nor on the choice of $i, j$ and $k$.

Let $\sigma$ denote the number of common neighbors in $\mathcal{P}_{k}$ of $x$ in $\mathcal{P}_{i}$ and $y$, adjacent to $x$, in $\mathcal{P}_{j}(i \neq j \neq k \neq i)$. Let $\tau$ denote the same parameter for $x$ and $y$ non-adjacent in $G$.

With the order $k-\lambda$ of the designs denoted by $n$, one can show that we may always assume that

$$
\sigma=\frac{1}{v}\left(k^{2}-\sqrt{n}(v-k)\right), \tau=\frac{k}{v}(k+\sqrt{n}) .
$$

Note that this fixes $\tau-\sigma$ to be $\sqrt{n}$, and we can not assume $2 k<v$ as is customary in design theory.

Assume now that $G$ is a linked system of $\ell$ symmetric $(v, k, \lambda)$ designs. Since $\sigma$ is an integer, $\ell>1$ implies that the order $n$ is a perfect square. We then obtain a 3-class $Q$-antipodal association scheme $(X, \mathbf{B})$ with associate matrices

$$
B_{0}=I, \quad B_{1}=A(\tilde{G}), \quad B_{2}=A\left(G_{2}\right), \quad B_{3}=A(G)
$$

where $G_{2}$ is the union of the $\ell+1$ complete graphs on the $Q$-antipodal classes $\mathcal{P}_{i}$ and $\tilde{G}$ is the multipartite complement of $G$; this is a linked system of $\ell$ symmetric $(v, v-k, v-2 k+\lambda)$ designs. We will not need the parameters of this association scheme with the exception of the Krein array: $\iota^{*}(X, \mathbf{B})=\left\{b_{0}^{*}, b_{1}^{*}, b_{2}^{*} ; c_{1}^{*}, c_{2}^{*}, c_{3}^{*}\right\}$ where

$b_{0}^{*}=c_{3}^{*}=m=v-1, \quad b_{1}^{*}=\ell c_{2}^{*}=\frac{\ell}{\ell+1}\left(v-2+\frac{1}{\sqrt{n}}(v-2 k)\right), \quad b_{2}^{*}=c_{1}^{*}=1$.

Such an association scheme is $Q$-antipodal and is not metric unless $\ell=1$.

Let $\mathbf{B}=\left\{B_{0}, B_{1}, B_{2}, B_{3}\right\}$ be the associate matrices of the association scheme arising from a linked system of symmetric designs on vertex set $X$ with parameters

$$
v=16 s^{2}, \quad n=4 s^{2}, \quad k=2 s(4 s-1), \quad \lambda=2 s(2 s-1)
$$

where $s$ is a positive integer. (From above, for $\ell>1$, we need the order $n$ to be a square. We will need $v=2 k+2 \sqrt{n}$ in order to ensure $b_{1}^{*}+c_{2}^{*}=m+1$. So our design must satisfy $v=4 n$ and $n$ must be even for $p_{11}^{1}$ to be integral when $\ell$ is even.) 
Theorem 3.6. Let $(X, \mathbf{B})$ be a linked system of symmetric designs with parameters $v=16 s^{2}, k=2 s(4 s-1)$ and $\lambda=2 s(2 s-1)$ and let $Y=X \times\{0,1\}$. Consider $\mathbf{A}=$ $\left\{A_{0}=I, A_{1}, A_{2}, A_{3}, A_{4}\right\}$ given by

$$
A_{1}=\left[\begin{array}{ll}
B_{3} & B_{1} \\
B_{1} & B_{3}
\end{array}\right], \quad A_{2}=\left[\begin{array}{ll}
B_{2} & B_{2} \\
B_{2} & B_{2}
\end{array}\right], \quad A_{3}=\left[\begin{array}{ll}
B_{1} & B_{3} \\
B_{3} & B_{1}
\end{array}\right], \quad A_{4}=\left[\begin{array}{ll}
0 & I \\
I & 0
\end{array}\right] .
$$

Then $(Y, \mathbf{A})$ is a 4-class $Q$-antipodal $Q$-bipartite cometric association scheme.

Proof: This is the extended $Q$-bipartite double of the scheme $(X, \mathbf{B})$, which is easily seen to satisfy the conditions of Theorem 3.1. So it remains only to check that the scheme is again $Q$-antipodal.

It is straightforward to verify that the intersection numbers are as follows: with $L_{h}=\left[p_{h j}^{i}\right]_{i, j}$, we have $L_{0}=I$,

$$
\begin{aligned}
& L_{1}=\left[\begin{array}{ccccc}
0 & \ell v & 0 & 0 & 0 \\
1 & 2(\ell-1) s(4 s+1) & v-1 & 2(\ell-1) s(4 s-1) & 0 \\
0 & 2 \ell n & 0 & 2 \ell n & 0 \\
0 & 2(\ell-1) s(4 s-1) & v-1 & 2(\ell-1) s(4 s+1) & 1 \\
0 & 0 & 0 & \ell v & 0
\end{array}\right], \\
& L_{2}=\left[\begin{array}{ccccc}
0 & 0 & 2(v-1) & 0 & 0 \\
0 & v-1 & 0 & v-1 & 0 \\
1 & 0 & 2(v-2) & 0 & 1 \\
0 & v-1 & 0 & v-1 & 0 \\
0 & 0 & 2(v-1) & 0 & 0
\end{array}\right] \\
& L_{3}=\left[\begin{array}{ccccc}
0 & 0 & 0 & \ell v & 0 \\
0 & 2(\ell-1) s(4 s-1) & v-1 & 2(\ell-1) s(4 s+1) & 1 \\
0 & 2 \ell n & 0 & 2 \ell n & 0 \\
1 & 2(\ell-1) s(4 s+1) & v-1 & 2(\ell-1) s(4 s-1) & 0 \\
0 & \ell v & 0 & 0 & 0
\end{array}\right] \text {, } \\
& L_{4}=\left[\begin{array}{lllll}
0 & 0 & 0 & 0 & 1 \\
0 & 0 & 0 & 1 & 0 \\
0 & 0 & 1 & 0 & 0 \\
0 & 1 & 0 & 0 & 0 \\
1 & 0 & 0 & 0 & 0
\end{array}\right]
\end{aligned}
$$

The Krein array is $\iota^{*}(Y, \mathbf{A})=\left\{v, v-1, \ell \frac{v}{\ell+1}, 1 ; 1, \frac{v}{\ell+1}, v-1, v\right\}$ which is both $Q$-bipartite and $Q$-antipodal.

One infinite family of linked systems of symmetric designs is known [5]. A description of these linked systems based on the Kerdock codes is cited in [5] as private 
communication from J. M. Goethals. The first published description is based on the "Cameron-Seidel scheme" [6]. Our presentation is based on [6] as well as [4].

The Cameron-Seidel scheme corresponds to a system of $\ell=2^{2 t+1}-1$ linked symmetric $\left(2^{2 t+2}, 2^{2 t+1}-2^{t}, 2^{2 t}-2^{t}\right)$ designs, where $t$ can be any positive integer. So, by deleting $Q$-antipodal classes, we obtain a system of $\ell$ such linked designs for any $\ell<2^{2 t+1}$. Each of these systems has order $n=k-\lambda=2^{2 t}$ an even square and $v=4 n$. So the construction given in Theorem 3.6 applies and we have an infinite family of 4-class $Q$-antipodal $Q$-bipartite cometric association schemes with $s=2^{t-1}$ in the language above.

We note that the Krein parameters are easily computed from the second eigenmatrix $Q$. Here, we give $Q$ and only the matrix of Krein parameters $L_{1}^{*}=\left[q_{1 j}^{k}\right]_{k, j}$ corresponding to the first idempotent in the $Q$-polynomial ordering:

$$
\begin{aligned}
Q & =\left[\begin{array}{ccccc}
1 & v & (\ell+1)(v-1) & \ell v & \ell \\
1 & 2^{t+1} & 0 & -2^{t+1} & -1 \\
1 & 0 & -\ell-1 & 0 & \ell \\
1 & -2^{t+1} & 0 & 2^{t+1} & -1 \\
1 & -v & (\ell+1)(v-1) & -\ell v & \ell
\end{array}\right], \\
L_{1}^{*} & =\left[\begin{array}{ccccc}
0 & v & 0 & 0 & 0 \\
1 & 0 & v-1 & 0 & 0 \\
0 & \frac{v}{\ell+1} & 0 & \frac{\ell v}{\ell+1} & 0 \\
0 & 0 & v-1 & 0 & 1 \\
0 & 0 & 0 & v & 0
\end{array}\right] .
\end{aligned}
$$

An important feature to note is that, unless $\ell+1$ divides $v$, the Krein parameter $c_{2}^{*}=q_{11}^{2}$ is non-integral. So these schemes cannot be duals of metric schemes in general. Moreover, for $\ell>1$, the schemes cannot be metric since they are $Q$-antipodal with more than two $Q$-antipodal classes.

\section{Structure of imprimitive cometric schemes}

In this section, we explore the structure of imprimitive cometric association schemes, treating the $Q$-bipartite case first and then the $Q$-antipodal case.

\subsection{Basic results for the $Q$-bipartite case}

A bipartite distance-regular graph obviously has $w=2$ bipartite halves; the next theorem is dual to this. In this proof, we consider the mapping $u_{1}$ which projects each elementary basis vector (or vertex) orthogonally into the eigenspace $V_{1}=\operatorname{colsp}\left(E_{1}\right)$, the column space of $E_{1}$. 
Theorem 4.1 ([3]). If (X, A) is Q-bipartite with $w$ dual bipartite classes of size $r$ each, then $r=2$.

Proof: The entries of the second eigenmatrix satisfy $Q_{i 2}=f\left(Q_{i 1}\right)$ where $f(t)=$ $\frac{1}{c_{2}^{*}}\left(t^{2}-a_{1}^{*} t-m\right)$. But $a_{1}^{*}=0$, so this is an even polynomial. Moreover, since $2 \in \mathcal{J}$, we must have $Q_{i 2}=m_{2}$ for all relations $i \in \mathcal{I}$. But the only solutions to this equation are $Q_{i 1}= \pm m$. So, assuming the natural ordering on relations, we must have $\mathcal{I}=$ $\{0, d\}$. The desired result $r=2$ will now follow from showing that $k_{d}=1$.

Let $x \in X$. Recall that the $(x, y)$-entry of $E_{1}$ is $\frac{m}{v} \cos (\theta)$ where $\theta$ is the angle formed by $u_{1}(x)$ and $u_{1}(y)$. If $\hat{x}$ is $d$-related to $x$, then clearly $u_{1}(\hat{x})=-u_{1}(x)$. The matrix $E_{1}$ has no repeated columns (since it generates $\mathcal{A}$ under Schur products) so this vector $\hat{x}$ is unique and $k_{d}=1$.

A special case of this result has been known for some time: a $Q$-polynomial antipodal distance-regular graph must be a double cover of its folded graph [2, Theorem 8.2.4].

Still assuming the natural ordering on relations, in the $Q$-bipartite case with vertices ordered so that dual bipartite pairs appear consecutively, this gives $A_{0}+A_{d}=I_{v / 2} \otimes$ $J_{2}$. Moreover, the dual bipartite classes are mapped by $u_{1}$ to opposing points on lines through the origin in $V_{1}$ (or in $\mathbb{R}^{m}$ ). This observation gives us the following two corollaries.

Corollary 4.2. Let $(X, \mathrm{~A})$ be a $Q$-bipartite cometric association scheme with the natural ordering on relations. Then, for the first eigenspace, the sequence $m=Q_{01}>$ $Q_{11}>\cdots>Q_{d 1}$ is symmetric about the origin. In particular, $Q_{\frac{d}{2}, 1}=0$ whenever $d$ is even.

Corollary 4.3. Let $(X, \mathrm{~A})$ be a $Q$-bipartite cometric association scheme with natural ordering on relations. Then the intersection numbers satisfy

$$
p_{i j}^{k}=p_{i, d-j}^{d-k}
$$

for all $0 \leq i, j, k \leq d$.

Question. Let $\rho: X \rightarrow \mathbb{R} P^{m-1}$ be a representation of the vertex set $X$ of a cometric association scheme $(X, \mathbf{A})$ by lines through the origin in $\mathbb{R}^{m}$ in such a way that the angle between lines $\rho(x)$ and $\rho(y)$ depends only on the relation joining $x$ and $y$ in $(X, \mathbf{A})$. What additional properties on $\rho$ imply the existence of a $Q$-bipartite double cover of the scheme?

\subsection{The $Q$-antipodal case and dismantlability}

Next, we move to the $Q$-antipodal case. In the study of linked systems of symmetric designs, one is interested in maximizing the parameter $\ell$ for given feasible values of $v, k$ and $\lambda$. More generally, we seek an upper bound on the number of $Q$-antipodal classes. We believe that the first multiplicity in the $Q$-polynomial ordering is a valid 
upper bound, as we now prove in the case where $d$ is odd. The dual result is due to Gardiner (cf. [2, Prop. 4.2.2]): for any antipodal distance-regular graph of valency $k$, the index $r$ of the cover is bounded above by $k$.

Theorem 4.4. Let $(X, \mathrm{~A})$ be a d-class $Q$-antipodal association scheme with $w$ $Q$-antipodal classes of size r each. If $d$ is odd, then $w \leq m$. If $d$ is even, then $w \leq m_{2}$.

Proof: From Theorem 2.2 and Theorem 2.1(iv), it is easy to deduce that

$$
E_{d}=\frac{1}{v}\left[\begin{array}{cccc}
(w-1) J & -J & \cdots & -J \\
-J & (w-1) J & \cdots & -J \\
\vdots & \vdots & \ddots & \vdots \\
-J & -J & \cdots & (w-1) J
\end{array}\right]
$$

so that $m_{d}=\operatorname{rank} E_{d}=w-1$. Using $b_{j}^{*}=c_{d-j}^{*}$ for $j \neq\left\lfloor\frac{d}{2}\right\rfloor$ and $b_{j}^{*} m_{j}=c_{j+1}^{*} m_{j+1}$, we then find $m_{d-j}=(w-1) m_{j}$ for $j<d / 2$.

First consider the case where $d$ is odd. Let $j=\frac{d-1}{2}$. Obviously,

$$
E_{1} \circ E_{j}=\frac{1}{v}\left[b_{j-1}^{*} E_{j-1}+a_{j}^{*} E_{j}+c_{j+1}^{*} E_{j+1}\right]
$$

and $b_{j-1}^{*}, c_{j+1}^{*}>0$. So the Absolute Bound gives

$$
(w-1) m_{\frac{d-1}{2}}=m_{\frac{d+1}{2}}<m_{\frac{d-3}{2}}+m_{\frac{d+1}{2}} \leq m m_{\frac{d-1}{2}} \Rightarrow w-1<m \Rightarrow w \leq m .
$$

Now if $d$ is even, we at least obtain $w \leq m_{2}$ by applying the same analysis to the Schur product $E_{2} \circ E_{\frac{d}{2}-1}$.

If we assume the natural ordering on relations, then a standard result about Sturm sequences tells us that the final column of $Q$ has $d$ sign changes. But we just saw that there are only two distinct entries in this column, namely $w-1$ and -1 . The relations in $\mathcal{I}$ are therefore $0,2,4, \ldots$. From this, we obtain the following two corollaries.

Corollary 4.5. In any $d$-class $Q$-antipodal scheme, $\left\lfloor\frac{d}{2}\right\rfloor$ non-trivial relations occur between vertices in the same $Q$-antipodal class and $\left\lceil\frac{d}{2}\right\rceil$ relations occur between classes. Namely, for $i$ odd, the partition into $Q$-antipodal classes is a proper vertex coloring of graph $G_{i}$ and for $i$ even, each component of $G_{i}$ lies entirely within some $Q$-antipodal class.

As $Q$-antipodal schemes include cometric bipartite distance-regular graphs, we expect their intersection numbers to behave in a manner similar to that observed for bipartite graphs. 
Corollary 4.6. Let $(X, \mathbf{A})$ be a $Q$-antipodal cometric association scheme with relations ordered naturally. Then the intersection numbers satisfy

$$
p_{i j}^{k}=0
$$

unless either $i+j+k$ is even or ijk is odd.

A $Q$-antipodal cometric association scheme with $Q$-antipodal classes $X_{1}, \ldots, X_{w}$ is dismantlable if, for any proper subset $\left\{X_{i_{1}}, \ldots, X_{i_{w^{\prime}}}\right\}$ of its $Q$-antipodal classes the set of induced graphs $\left(G_{i}\right)_{Y \times Y}$ where $0 \leq i \leq d$ and $Y=X_{i_{1}} \cup \cdots \cup X_{i_{w^{\prime}}}$ is again an association scheme. For $w^{\prime}=1$, it is a standard result (originally due to Rao, Ray-Chaudhuri and Singhi-see [2, Section 2.4]) that we find a subscheme on each $X_{i}$ and these all have the same parameters. (Here, we call this the local scheme.)

Theorem 4.7. Every $Q$-antipodal scheme is dismantlable. The subscheme induced on any non-trivial collection of $w^{\prime} Q$-antipodal classes is cometric for $w^{\prime} \geq 1$ and $Q$-antipodal with d classes for $w^{\prime}>1$.

Proof: Since any $Q$-antipodal class $X_{i}$ has dual distribution ([2, p. 333]) $a Q=$ $[r, 0, \ldots, 0, r(w-1)]$ it has strength $t=d-1$ as a Delsarte design. From Corollary 4.5, $X_{i}$ has degree $s=\left\lfloor\frac{d}{2}\right\rfloor$, so the result follows immediately in the case $w^{\prime}=1$ using Delsarte's Theorem [9, Theorem 5.25] which now implies that the local scheme is cometric.

Now suppose, without loss, that $Y=X_{1} \cup \cdots \cup X_{w^{\prime}}$ for some $1<w^{\prime}<w$. We apply the technique of Delsarte (cf. [2, p. 61-62]) to show that the submatrices $\bar{E}_{j}$ of $E_{j}$ obtained by restricting to rows and columns indexed by vertices in $Y$ span a Bose-Mesner algebra.

Clearly the vector space $\overline{\mathcal{A}}:=\{\bar{M}: M \in \mathcal{A}\}$ of real symmetric matrices is closed under entrywise multiplication and contains the identity and all-ones matrices. So, by the classic theorem of Bose and Mesner, it remains to prove that $\overline{\mathcal{A}}$ is closed under matrix multiplication.

The dual distribution of $Y$ has entries $(a Q)_{0}=r w^{\prime},(a Q)_{d}=r\left(w-w^{\prime}\right)$ and $(a Q)_{j}=0$ for $j \neq 0, d$. Applying Eq. (9) on page 61 of [2], we then have

$$
\left\|v E_{k} \Delta_{Y} E_{\ell}-|Y| \delta_{k, \ell} E_{k}\right\|^{2}=q_{k, \ell}^{d} r^{2} w^{\prime}\left(w-w^{\prime}\right)
$$

for $0 \leq k, \ell \leq d$ where $\Delta_{Y}$ is the diagonal matrix with a one in position $y, y$ for $y \in Y$ and zeros elsewhere and $\|\cdot\|$ denotes the Hermitian norm. Since $\bar{E}_{k} \bar{E}_{\ell}$ is a submatrix of $E_{k} \Delta_{Y} E_{\ell}, q_{k, \ell}^{d}=0$ implies $\bar{E}_{k} \bar{E}_{\ell}=0$ for $k \neq \ell$ and $q_{k, k}^{d}=0$ implies $\bar{E}_{k} \bar{E}_{k}=|Y| \bar{E}_{k}$. Since $(X, \mathbf{A})$ is $Q$-antipodal, we have $q_{k, \ell}^{d}=0$ unless $k+\ell=d$ or $k=\ell \geq d / 2$. So it remains to prove that $\bar{E}_{k} \bar{E}_{\ell}$ belongs to $\overline{\mathcal{A}}$ in these cases.

For $k \leq d / 2$, we observe that $\sum_{j} \bar{E}_{j}=I$ and $\bar{E}_{k} I$ belongs to $\overline{\mathcal{A}}$ showing that

$$
\bar{E}_{k} \bar{E}_{d-k}=\bar{E}_{k}\left(I-\sum_{j \neq d-k} \bar{E}_{j}\right)
$$


also belongs to $\overline{\mathcal{A}}$. Now the same trick works for $k>d / 2$ : we have just established that $\bar{E}_{k} \bar{E}_{d-k}$ lies in $\overline{\mathcal{A}}$ so we may conclude that

$$
\bar{E}_{k} \bar{E}_{k}=\bar{E}_{k}\left(I-\sum_{j \neq k} \bar{E}_{j}\right)
$$

belongs to $\overline{\mathcal{A}}$ as well. Thus $\overline{\mathcal{A}}$ is a Bose-Mesner algebra.

The Krein parameters are, up to a positive scalar multiple, the same as those for the original scheme. So $\bar{E}_{0}, \bar{E}_{1}, \ldots, \bar{E}_{d}$ is a $Q$-polynomial ordering. The partition $\left\{X_{1}, \ldots, X_{w^{\prime}}\right\}$ is clearly an imprimitivity system and, using Suzuki's Theorem, we conclude that the subscheme is $Q$-antipodal for $w^{\prime}>1$.

Remark 4.8. We can view this theorem as formally dual to an unpublished result of Godsil (personal communication) which states that, in an antipodal distance-regular graph, any subset of an antipodal class is a completely regular code. While this does not in itself guarantee the existence of a quotient scheme, see [2, Remark (iv), p. 385] for an interesting special case due to Hensel in which certain partitions of the antipodal classes into completely regular codes give rise to quotient distance-regular graphs which are also antipodal.

Example 4.9. The 24-cell in $\mathbb{R}^{4}$ is an instructive example. There are three $Q$-antipodal classes of size eight and, in the polytope, the convex hull of any two of them yields a Euclidean 4-cube.

Since there exist antipodal distance-regular graphs with index $r=2$ which are not $Q$-polynomial (e.g., the dodecahedron), we expect there to be $Q$-antipodal schemes with $w=2$ which are not metric. Indeed, consider the following examples.

Example 4.10. The coset graph of the shortened ternary Golay code, labeled (A17) in [2, p. 365] has intersection array $\{20,18,4,1 ; 1,2,18,20\}$; this is an antipodal distance-regular graph belonging to a translation scheme. The dual association scheme is $Q$-antipodal on $v=243$ vertices with $w=3 Q$-antipodal classes. Removing one of these, we obtain a $Q$-antipodal scheme on 162 vertices having $w=2 Q$-antipodal classes which is not metric. Note that this scheme has parameters

$$
d=4, v=162, \iota^{*}(X, \mathbf{A})=\{20,18,3,1 ; 1,3,18,20\}
$$

formally dual to those of an unknown diameter four bipartite distance-regular graph, but it is not realizable as a translation scheme.

Example 4.11. The same idea applied to graphs labeled (A16) and (A18) on page 365 of [2] yield new $Q$-antipodal schemes with parameters

$$
\begin{aligned}
& d=5, v=486, \iota^{*}(X, \mathbf{A})=\left\{22,20, \frac{27}{2}, 2,1 ; 1,2, \frac{27}{2}, 20,22\right\}, w=2 \\
& d=6, v=1536, \iota^{*}(X, \mathbf{A})=\{21,20,16,8,2,1 ; 1,2,4,16,20,21\}, w=3 .
\end{aligned}
$$

On the same page of [2], the dual of this last scheme is ruled out. 
We briefly mention some more special cases of Theorem 4.7. A $Q$-polynomial distance-regular graph is $Q$-antipodal if and only if it is bipartite. The $Q$-antipodal classes are precisely the $w=2$ bipartite color classes. The induced configuration on either one of these classes has long been known to be a cometric association scheme (see [2, Prop. 4.2.2] and preceding discussion). So the theorem is trivial in the metric case. It clearly also holds (by definition) for any linked system of symmetric designs and therefore also for the new family of 4-class schemes introduced in Section 3.1. For an arbitrary $Q$-antipodal 3-class scheme, we can take $w^{\prime}=3$ to obtain a new proof of the following result, which was proved by Haemers in the case $(v, k, \lambda)=(16,6,2)$ :

Corollary 4.12 (van Dam [8, Theorem 5.8]). Every Q-antipodal 3-class cometric association scheme arises from a linked system of symmetric designs.

Question. Is there an analogous characterization of 4-class cometric schemes which are both $Q$-bipartite and $Q$-antipodal?

The octahedron is a $Q$-antipodal scheme with $r=2$ and $w=3$. In this case, $m=3$ while $m=2$ for any induced subscheme on $w^{\prime}=2 Q$-antipodal classes. In spite of this, for $Q$-antipodal schemes with three or more classes, these two multiplicities coincide.

Theorem 4.13. For a $Q$-antipodal $d$-class association scheme with $d \geq 3$ and $w$ $Q$-antipodal classes of size $r$, the first multiplicity $m$ does not depend on $w$ but only on the parameters of the local scheme.

Proof: Let $\bar{E}_{j}$ denote the submatrix of $E_{j}$ with rows and columns restricted to one $Q$-antipodal class $X_{i}$. Let $r_{j}=\operatorname{rank} \bar{E}_{j}$. Using results of Delsarte (see the proofs of Theorem 5.25 and Corollary 5.26 in [9]), we know that $\bar{E}_{j}$ is a positive scalar multiple of the $j$ th primitive idempotent in the $Q$-polynomial ordering for the local scheme. It is also well-known that the local schemes all have the same parameters, so $r_{j}$ is independent of $X_{i}$.

If $j<d / 2$, then $E_{j}+E_{d-j}$ is a block-diagonal matrix the blocks of which all have the same $\operatorname{rank} r_{j}$. Hence $\operatorname{rank}\left(E_{j}+E_{d-j}\right)=w r_{j}$. On the other hand,

$$
\operatorname{rank}\left(E_{j}+E_{d-j}\right)=m_{j}+m_{d-j}=m_{j}+m_{d} m_{j}=w m_{j} .
$$

So $r_{j}=m_{j}$ for all $j<d / 2$, and $\operatorname{rank} \bar{E}_{1}=m$ as desired.

Many examples of $Q$-antipodal schemes are known with $w<m$; the natural question arises as to whether these can be extended to $Q$-antipodal cometric schemes with larger $w$. We now see, at least when $d$ is odd, that the number of fibers in the larger scheme is bounded above by the first multiplicity in the given scheme. So, for example, if we look at any bipartite $Q$-polynomial distance-regular graph, we can ask if it can be extended to a larger $Q$-antipodal scheme with $w>2$. (E.g., as we have seen, this is possible for the $n$-cube for $n=2,4$ and degenerately for $n=3$.) For $d$ odd, an upper bound on $w$ is the multiplicity of the first eigenvalue in the $Q$-polynomial ordering for the bipartite graph. 
Having said this, we expect schemes with $w=m$ to be extremely rare and to have very nice structure. By analogy, each antipodal $(n-1)$-fold cover of the complete graph $K_{n}$ gives rise to a Moore graph of valency $n$ and diameter two [12] and any antipodal $k$-fold cover of a complete bipartite graph $K_{k, k}$ yields an affine plane. We know of no other antipodal distance-regular graphs with $r=k$. Thus it is natural to expect stronger bounds on $w$ unless the parameters are particularly nice.

Indeed, bounds much better than that given in Theorem 4.4 are known in the case of linked systems of symmetric designs (i.e., $d=3$ ). For example, we have the trivial bound $w \leq 2$ when $n$ is not a square. Moreover, since $m_{2}=(w-1) m$ in this case, we obtain

$$
1+m_{2}=1+(w-1) m \leq \operatorname{rank}\left(E_{1} \circ E_{1}\right) \leq \frac{1}{2} m(m+1)
$$

giving $w \leq \frac{m+2}{2}$. Mathon [14] and Noda [16] give stronger bounds for the number of linked symmetric $(v, k, \lambda)$ designs, but only in the case when the quantity $(\sigma-\tau)(v-$ $2 k$ ) is positive. For example, their bounds do not apply to the case $v=36, n=9$.

In the spirit of the Bannai-Ito Conjecture mentioned in our introduction, we expect $\lim _{d \rightarrow \infty} w=2$.

Acknowledgments Initial work on some of the questions in the latter part of this paper was carried out by WJM in collaboration with Dom de Caen who urged patience in the search for constructions. That was in 1999. The association schemes constructed in Section 3 were discovered in response to a challenge by Chris Godsil to find more cometric schemes that are neither metric nor duals of metric schemes. Eiichi Bannai and Akihiro Munemasa improved an earlier draft list of known cometric schemes, and the anonymous referees suggested numerous improvements.

\section{References}

1. E. Bannai and T. Ito, Algebraic Combinatorics I: Association Schemes, Benjamin-Cummings, Menlo Park, 1984.

2. A.E. Brouwer, A.M. Cohen, and A. Neumaier, Distance-Regular Graphs, Springer-Verlag, Berlin, 1989.

3. A.E. Brouwer, C.D. Godsil, J.H. Koolen, and W.J. Martin, "Width and dual width of subsets in metric and cometric association schemes," J. Combin. Th. Ser. A 102 (2003), 255-271.

4. D. de Caen and E.R. van Dam, "Association schemes related to Kasami codes and Kerdock sets," Des. Codes Cryptogr. 18 (1999), 89-102.

5. P.J. Cameron, "On groups with several doubly-transitive permutation representations," Math. Z. 128 (1972), 1-14.

6. P.J. Cameron and J.J. Seidel, "Quadratic forms over GF(2)," Proc. Koninkl. Nederl. Akademie van Wetenschappen, Series A, Vol. 76 Indag. Math. 35 (1973), 1-8.

7. D. Cerzo and H. Suzuki, "On imprimitive $Q$-polynomial schemes of exceptional type," Preprint, 2006.

8. E. van Dam, "Three-class association schemes," J. Alg. Combin. 10(1) (1999), 69-107.

9. P. Delsarte, "An algebraic approach to the association schemes of coding theory," Philips Res. Repts. Suppl. 10 (1973).

10. P. Delsarte, J.-M. Goethals, and J.J. Seidel, "Spherical codes and designs," Geom. Ded. 6 (1977), 363-388.

11. G.A. Dickie, “ $Q$-polynomial structures for association schemes and distance-regular graphs,” Ph.D. Thesis, University of Wisconsin-Madison, 1995.

12. A.D. Gardiner, “Antipodal covering graphs," J. Combin. Th. Ser. B 16 (1974), 255-273.

Springer 
13. C.D. Godsil, Algebraic Combinatorics, Chapman and Hall, New York, 1993.

14. R. Mathon, "The systems of linked 2-(16,6,2) designs," Ars Comb. 11 (1981), 131-148.

15. A. Munemasa, "Spherical designs," in Handbook of Combinatorial Designs (2nd ed.), C.J. Colbourn and J.H. Dinitz (eds.), CRC Press, Boca Raton, 2006, Section VI.54, pp. 637643.

16. R. Noda, "On homogeneous systems of linked symmetric designs," Math. Z. 138 (1974), 15-20.

17. H. Suzuki, "Imprimitive $Q$-polynomial association schemes," J. Alg. Combin. 7(2) (1998), 165180 .

18. P. Terwilliger, "The subconstituent algebra of an association scheme I," J. Alg. Combin. 1(4) (1992), $363-388$. 